\title{
A new mCRE-DDM based approach for model updating in structural dynamics with industrial applications
}

\author{
Z. Samir ${ }^{*, 1,2}$, L. Chamoin ${ }^{1}$ and M. Abbas ${ }^{2,3}$ \\ ${ }^{1}$ Université Paris-Saclay, ENS Paris-Saclay, CNRS, LMT \\ 4 Avenue des Sciences, 91190 Gif-sur-Yvette, France \\ ${ }^{2}$ EDF R\&D - Dept. ERMES \\ 7 Boulevard Gaspard Monge, 91120 Palaiseau, France \\ ${ }^{3}$ IMSIA - UMR 9219 / EDF/CNRS/CEA/ENSTA \\ 828 Boulevard des Maréchaux, 91762 Palaiseau Cedex, France \\ * Corresponding author \\ E-mail : zouhair.samir@edf.fr
}

\begin{abstract}
The modified constitutive relation error (mCRE) has been widely used for validating and updating numerical models in structural mechanics [1], since it offers some advantageous properties such as convexity, modeling errors localisation and robustness to noisy measurements. Inverse problems based on this functional are classically solved using a two-steps iterative process. Each iteration begins with a step of localization of erroneous regions of the structure, followed by a correction step of concerned parameters. The localization step consists of solving a large system of equations which needs to be reassembled after each correction step, since the underlying parameters of the system change. This implies an important computational time which present a real bottleneck of the mCRE based methods and make them less convenient for problems of industrial size.
\end{abstract}

To overcome this problem, we propose an approach based on domain decomposition techniques [2] which are suited to the mCRE localisation step. Our approach benefits from the same advantages of DDM methods such as parallelization which allows dealing with large problems in a reasonable computational time. Moreover, the correction step can be performed in parallel for independent subdomains and only those whose parameters have changed need to reassemble their part of the problem. Besides these numerical advantages, the new approach allows changing a subdomain model easily which makes it more flexible.

In this context, we developed a primal and a dual variant of this approach inspired respectively by the classical BDD and FETI methods. Adapted preconditioners and coarse problems are also proposed. The approach will be illustrated and performance will be evaluated on several numerical experiments.

\section{REFERENCES}

[1] A. Chouaki, P. Ladevèze and L. Proslier, "An updating of structural dynamic model with damping", Inverse Problems in Engineering: Theory and Practice, pp. 335-342, (1996).

[2] P. Gosselet and C. Rey, "Non-overlapping domain decomposition methods in structural mechanics", Archives of Computational Methods in Engineering, Vol. 13, pp. 515-572, (2006). 